\title{
Phenomenology of the Zee model for Dirac neutrinos and general neutrino interactions
}

\author{
Julián Calle $\odot,{ }^{*}$ Diego Restrepo ${ }^{\dagger}{ }^{\dagger}$ and Óscar Zapata $\odot^{\ddagger}$ \\ Instituto de Física, Universidad de Antioquia, Calle 70 No. 52-21, \\ Apartado Aéreo 1226, Medellín, Colombia
}

(Received 8 April 2021; accepted 21 June 2021; published 28 July 2021)

\begin{abstract}
The Zee model for Dirac neutrinos is one of the simplest models featuring one-loop Dirac neutrino masses. The interactions between the new scalars (two singly charged fields) and neutrinos induce general neutrino interactions (GNI) which, as a generalization of the nonstandard neutrino interactions, constitute an additional tool to probe models beyond the Standard Model like this. In this work, we consider a $U(1)_{B-L}$ gauge symmetry as being responsible for the Diracness of the neutrinos and the radiative character of the neutrino masses. We determine the viable parameter space consistent with neutrino oscillation data, leptonic rare decays, and collider constraints, and establish the most relevant experimental prospects regarding lepton flavor violation searches and GNI in future solar neutrino experiments.
\end{abstract}

DOI: 10.1103/PhysRevD.104.015032

\section{INTRODUCTION}

After several decades of research dedicated to neutrino physics [1,2], the underlying mechanism behind the massiveness of neutrinos remains one of the unresolved conundrums of the Standard Model (SM). In addition to this, the yet-to-be determined question of whether neutrinos are different from their antiparticles [3] and the lack of signatures of lepton flavor violation (LFV) in the charged sector [4,5] make the landscape even more unclear. Fortunately, the very rich experimental program in the lepton sector is undoubtedly rather clear and promising, as for instance, due to the entrance in operation during the current decade of neutrino oscillation experiments such as JUNO [6], DUNE [7], Hyper-Kamiokande [8], among others, and the great experimental effort on searching for LFV with, in some cases, reaching a sensitivity improvement of several orders of magnitude [9-14].

The simplest way to generate neutrino masses is to add to the SM spectrum three right-handed neutrinos $\nu_{R i}$ coupled to the SM Higgs through a term in the Lagrangian as [15-19]

$$
\mathcal{L}_{4 \nu}=y_{D} \bar{L} . \tilde{H} \nu_{R}+\text { H.c., }
$$

\footnotetext{
*julian.callem@udea.edu.co restrepo@udea.edu.co

¥oalberto.zapata@udea.edu.co
}

Published by the American Physical Society under the terms of the Creative Commons Attribution 4.0 International license. Further distribution of this work must maintain attribution to the author(s) and the published article's title, journal citation, and DOI. Funded by SCOAP ${ }^{3}$. where $L$ is the lepton doublet, $H$ is the SM Higgs doublet, and $y_{D}$ is the matrix of neutrino Yukawa couplings. Since after the electroweak symmetry breaking (and assuming lepton number conservation) such a term leads to sub-eV neutrino Dirac masses for $\left|y_{D}\right| \lesssim 10^{-13}$, the explanation of the smallness of the neutrino mass scale is yet to be settled. A way out of this dilemma may be to forbid $\mathcal{L}_{4 \nu}$ by imposing a $U(1)_{B-L}$ local symmetry, but generate it at loop level via the five-dimensional operator [20-22]

$$
\mathcal{L}_{5 \nu}=y_{D}^{\prime} \bar{L} . \tilde{H} \nu_{R} . S+\text { H.c. },
$$

with $S$ being the singlet scalar field responsible for the breaking of the $B-L$ symmetry. From the huge variety of one-loop Dirac neutrino models, ${ }^{1}$ the one including only two singlet charged scalars and three singlet right-handed neutrinos [24-26] may be considered as the simplest one: we dub it the Zee model for Dirac neutrinos.

In this paper we consider the Zee model for Dirac neutrinos with a $B-L$ symmetry, a realization obtained following the approach discussed in Refs. [20,21,27,28]. Thanks to this new symmetry (with the charge assignment given in Table I), it is possible to forbid the tree-level contribution of $\mathcal{L}_{5 \nu}$ without any extra ad hoc symmetry. ${ }^{2}$ As occurs in the original Zee model [29,30], neutrino masses are generated at one loop with the SM charged leptons running inside the loop, which is closed thanks to a trilinear interaction term between the charged scalars and the Higgs field. Both charged singlets not only give rise to

\footnotetext{
${ }^{1}$ For a recent review, see Ref. [23].

${ }^{2}$ Notice that in Refs. [24,25] such a contribution is forbidden by a softly broken $Z_{2}$ symmetry.
} 
LFV processes and may leave signatures at the LHC, but also induce general neutrino interactions (GNI) [31-33]. We will determine the viable parameter space consistent with neutrino oscillation observables and LFV and LHC constraints, and we will establish the most relevant experimental perspectives regarding LFV searches and GNI in future solar neutrino experiments.

The rest of the paper is organized as follows. In Sec. II we present the model and discuss the neutrino mass generation, the LFV processes, and LHC signatures. In Sec. III we deduce the expressions for the general neutrino interactions, and the phenomenological analysis is presented in Sec. IV. Finally, we conclude in Sec. V.

\section{THE MODEL}

The realization of the Zee Dirac model through a $U(1)_{B-L}$ gauge symmetry and without new extra fermions besides the three right-handed neutrinos $\nu_{R j}$ requires the introduction of two $S U(2)_{L}$-singlet charged scalars, $\sigma_{1}^{ \pm}$and $\sigma_{2}^{ \pm}$, and the two bosonic fields associated with the new symmetry, $Z_{\mu}^{\prime}$ and $S$, as the gauge boson and the scalar responsible for the symmetry breaking, respectively. ${ }^{3}$ The new particle content is summarized in Table I, where the charge assignment for the leptons and right-handed neutrinos guarantees a successful anomaly cancellation [37,38] and protects the Diracness of neutrinos.

The most general Lagrangian contains the following Yukawa terms:

$$
-\mathcal{L} \supset f_{\alpha \beta} \overline{L_{\alpha}^{c}} L_{\beta} \sigma_{1}^{+}+h_{\beta j} \overline{\ell_{R \beta}^{c}} \nu_{R j} \sigma_{2}^{+}+\text {H.c. }
$$

TABLE I. Particle content with the electroweak and $U(1)_{B-L}$ quantum numbers. $i=1, j=2, k=3(i=3, j=2, k=1)$ correspond to a normal (inverted) neutrino mass ordering.

\begin{tabular}{lcrc}
\hline \hline Symbol & $\left(S U(2)_{L}, U(1)_{Y}\right)$ & $L$ & Spin \\
\hline$L$ & $(2,-1 / 2)$ & -1 & $1 / 2$ \\
$H$ & $(2,1 / 2)$ & 0 & 0 \\
$\overline{\ell_{R}}$ & $(1,1)$ & -1 & $1 / 2$ \\
$\overline{\nu_{R i, j}}$ & $(1,0)$ & 4 & $1 / 2$ \\
$\overline{\nu_{R k}}$ & $(1,0)$ & -5 & $1 / 2$ \\
$S$ & $(1,0)$ & 3 & 0 \\
$\sigma_{1}^{+}$ & $(1,1)$ & 2 & 0 \\
$\sigma_{2}^{+}$ & $(1,1)$ & 5 & 0 \\
\hline \hline
\end{tabular}

where $L_{\alpha}$ and $\ell_{R \alpha}$ are lepton doublets and singlets, respectively, $c$ is the charge-conjugation operator, $f_{\alpha \beta}$ and $h_{\beta j}$ are $3 \times 3$ matrices in flavor space, and $\alpha, \beta=1,2,3$ are family indices. The coupling $f$ is an antisymmetric complex matrix, ${ }^{4}$ whereas all of the nonzero $h_{\beta j}$ couplings are complex in general. Note that there are three vanishing couplings because the lepton number $(L)$ assignment of right-handed neutrino of $L$ charge $-5: \nu_{R 3}\left(\nu_{R 1}\right)$ for the case of a normal (inverted) hierarchy of neutrino masses. ${ }^{5}$ This massless chiral fermion can either contribute to the effective number of relativistic degrees of freedom $N_{\text {eff }}$ in the early Universe [20,22] or become massive and be a good Majorana dark matter candidate after the introduction of an extra singlet scalar $S^{\prime}$ of $L$ charge $-10[22,38]$. In the last case, the extra contribution to $N_{\text {eff }}$ comes from the Goldstone boson associated with the imaginary part of the complex singlet scalar field $S^{\prime}$ [22]. The introduction of the extra scalar fields leads to the scalar potential

$$
\begin{aligned}
\mathcal{V}\left(H, S, \sigma_{1}, \sigma_{2}\right)= & \mu_{H}^{2} H^{\dagger} H+\lambda_{H}\left(H^{\dagger} H\right)^{2}+\mu_{S}^{2} S^{\dagger} S+\lambda_{S}\left(S^{\dagger} S\right)^{2}+\mu_{1}^{2}\left|\sigma_{1}^{+}\right|^{2}+\mu_{2}^{2}\left|\sigma_{2}^{+}\right|^{2} \\
& +\left[\mu_{3} \sigma_{1}^{+} \sigma_{2}^{-} S+\text { H.c. }\right]+\lambda_{1}\left|\sigma_{1}^{+}\right|^{4}+\lambda_{2}\left|\sigma_{2}^{+}\right|^{4}+\lambda_{3}\left(H^{\dagger} H\right)\left(S^{\dagger} S\right)+\lambda_{4}\left|\sigma_{1}^{+}\right|^{2}\left|\sigma_{2}^{+}\right|^{2} \\
& +\lambda_{5}\left(H^{\dagger} H\right)\left|\sigma_{1}^{+}\right|^{2}+\lambda_{6}\left(H^{\dagger} H\right)\left|\sigma_{2}^{+}\right|^{2}+\lambda_{7}\left(S^{\dagger} S\right)\left|\sigma_{1}^{+}\right|^{2}+\lambda_{8}\left(S^{\dagger} S\right)\left|\sigma_{2}^{+}\right|^{2} .
\end{aligned}
$$

We assume $\mu_{H}^{2}<0, \mu_{S}^{2}<0$, and $\operatorname{Im}\left(\mu_{3}\right)=0$. Moreover, we assume $\lambda_{3} \ll 1$ such that the scalar $S$ and $H$ do not mix, allowing us to identify the $C P$-even scalar particle in $H$ as

\footnotetext{
${ }^{3}$ If such a breaking is associated with a strong first-order phase transition with the subsequent broadcasting of gravitational waves, it may be possible to further test this model in the planned gravitational-wave experiments (see Refs. [34-36] for related studies).

${ }^{4}$ Notice that by performing a field redefinition on $\sigma_{1}$ it is possible to make one of such couplings real.

${ }^{5}$ If only one charged scalar is added, then the right-handed and left-handed neutrinos would have the same charge under the $B-L$ symmetry, thus rendering the Yukawa interaction term $\bar{L}_{\alpha} \tilde{H} \nu_{R j}$ allowed.
}

the SM Higgs boson. To establish the scalar spectrum, we expand the scalar fields as

$$
H=\left(\begin{array}{c}
0 \\
\frac{1}{\sqrt{2}}\left(h+v_{H}\right)
\end{array}\right), \quad S=\frac{1}{\sqrt{2}}\left(S_{R}+v_{S}\right),
$$

with $v_{H}=246.22 \mathrm{GeV}$. Of the original ten scalar degrees of freedom in the model, the gauge bosons $W^{ \pm}$, $Z^{0}$, and $Z^{\prime}$ absorb four of them. Thus, the scalar spectrum contains two neutral $C P$-even states $\left(h\right.$ and $S_{R}$ ) and two charged scalars $\left(s_{1}^{ \pm}\right.$and $s_{2}^{ \pm}$). Mass eigenstates for new charged scalars are defined through the mixing angle $\varphi$ as 


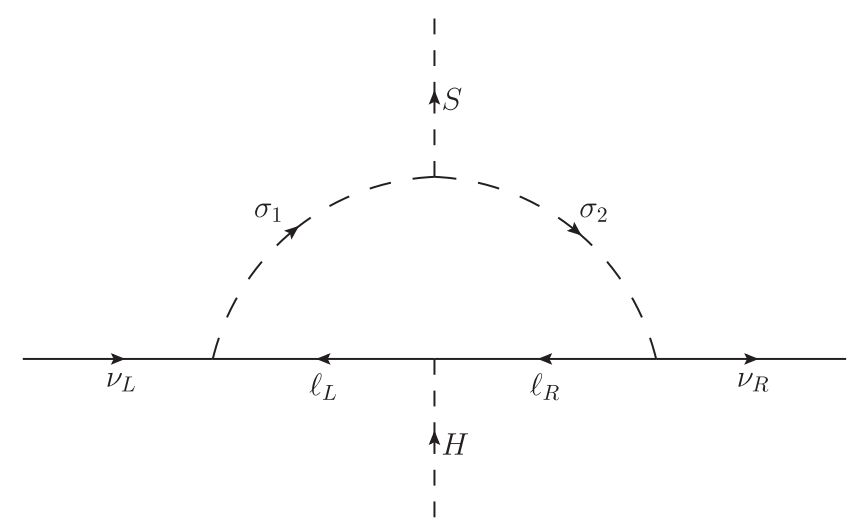

FIG. 1. $B-L$ flux in the one-loop diagram leading to Dirac neutrino masses.

$$
\left(\begin{array}{c}
s_{1}^{ \pm} \\
s_{2}^{ \pm}
\end{array}\right)=\left(\begin{array}{cc}
\cos \varphi & -\sin \varphi \\
\sin \varphi & \cos \varphi
\end{array}\right)\left(\begin{array}{c}
\sigma_{1}^{ \pm} \\
\sigma_{2}^{ \pm}
\end{array}\right),
$$

where the mixing angle is given by $\sin (2 \varphi)=\sqrt{2} \mu_{3} v_{S} /$ $\left(m_{s_{2}}^{2}-m_{s_{1}}^{2}\right)$. By defining $m_{\sigma_{1}}^{2}=\mu_{1}^{2}+\lambda_{5} v_{H}^{2} / 2+\lambda_{7} v_{S}^{2} / 2$, $m_{\sigma_{2}}^{2}=\mu_{2}^{2}+\lambda_{6} v_{H}^{2} / 2+\lambda_{8} v_{S}^{2} / 2$, their masses take the form

$m_{s_{1}, s_{2}}^{2}=\frac{1}{2}\left(m_{\sigma_{2}}^{2}+m_{\sigma_{1}}^{2} \mp \sqrt{\left(m_{\sigma_{2}}^{2}-m_{\sigma_{1}}^{2}\right)^{2}+8 \mu_{3}^{2} v_{S}^{2}}\right)$,

with $s_{1}^{ \pm}$being the lightest one. Since the effect of $\lambda_{5,6,7,8}$ on $m_{\sigma_{i}}^{2}$ can be absorbed by rescaling the $\mu_{i}^{2}$ parameters, hereafter we further assume $\lambda_{5,6,7,8} \ll 1$.

\section{A. Radiative neutrino masses}

The interplay of the new Yukawa interactions and the trilinear interaction $\mu_{3}$ generates Dirac neutrino masses at the one-loop level, as displayed in Fig. 1. The expression for the corresponding mass matrix can be cast as

$$
\left[M_{\nu}\right]_{\alpha i}=\kappa\left[f^{T}\right]_{\alpha \beta}\left[M_{\ell}\right]_{\beta \beta} h_{\beta i},
$$

where $M_{\ell}$ is the diagonal mass matrix of the charged leptons and

$$
\kappa=\frac{\sin (2 \varphi)}{16 \pi^{2}} \ln \frac{m_{s_{2}}^{2}}{m_{s_{1}}^{2}}
$$

$M_{\nu}$ is diagonalized by the biunitary transformation

$$
\left(U_{L}^{(\nu)}\right)^{\dagger} M_{\nu} U_{R}^{(\nu)}=M_{\nu}^{\mathrm{diag}}=\operatorname{diag}\left(m_{\nu_{1}}, m_{\nu_{2}}, m_{\nu_{3}}\right),
$$

where $U_{R}^{(\nu)}$ and $U_{L}^{(\nu)}$ are rotation $3 \times 3$ matrices associated with the right-handed and left-handed neutrinos, respectively. Assuming $U_{R}^{(\nu)}=\mathbb{1}$, the lepton mixing matrix takes the form

$$
U_{\mathrm{PMNS}}=U_{L}^{(\nu)}
$$

Due to the antisymmetric character of the Yukawa coupling matrix $f$ one neutrino state remains massless, and from Eq. (7) one can express six of the nine nonzero Yukawa couplings in terms of the neutrino oscillation observables, the masses of the charged leptons and scalars. The expressions for the nonzero couplings in the case of a normal neutrino mass hierarchy $(\mathrm{NH})$ are

$$
\begin{gathered}
h_{22}=-\frac{\left(U_{13} U_{32}-U_{12} U_{33}\right)\left(f_{13} h_{12} \kappa m_{e}-m_{2} U_{32}\right)}{f_{13} \kappa\left(U_{23} U_{32}-U_{22} U_{33}\right) m_{\mu}}, \\
h_{23}=\frac{\left(U_{12} U_{33}-U_{13} U_{32}\right)\left(f_{13} h_{13} \kappa m_{e}-m_{3} U_{33}\right)}{f_{13} \kappa\left(U_{23} U_{32}-U_{22} U_{33}\right) m_{\mu}}, \\
h_{32}=\frac{f_{13} h_{12} \kappa m_{e}\left(U_{13} U_{22}-U_{12} U_{23}\right)+m_{2} U_{22}\left(-U_{13} U_{32}+U_{12} U_{33}\right)}{f_{13} \kappa\left(U_{23} U_{32}-U_{22} U_{33}\right) m_{\tau}}, \\
h_{33}=\frac{f_{13} h_{13} \kappa m_{e}\left(U_{13} U_{22}-U_{12} U_{23}\right)+m_{3} U_{23}\left(-U_{13} U_{32}+U_{12} U_{33}\right)}{f_{13} \kappa\left(U_{23} U_{32}-U_{22} U_{33}\right) m_{\tau}}, \\
f_{12}=\frac{f_{13}\left(U_{13} U_{22}-U_{12} U_{23}\right)}{U_{13} U_{32}-U_{12} U_{33}}, \\
f_{23}=\frac{f_{13}\left(U_{23} U_{32}-U_{22} U_{33}\right)}{U_{13} U_{32}-U_{12} U_{33}},
\end{gathered}
$$

with $U_{i j}=\left(U_{\mathrm{PMNS}}\right)_{i j}, m_{2}=\sqrt{\Delta m_{\mathrm{sol}}^{2}}$, and $m_{3}=\sqrt{\Delta m_{\mathrm{atm}}^{2}}$. The expressions for the case of an inverted neutrino mass hierarchy (IH) are 


$$
\begin{gathered}
h_{22}=\frac{\left(U_{11} U_{32}-U_{12} U_{31}\right)\left(f_{13} h_{12} \kappa m_{e}-m_{2} U_{32}\right)}{f_{13} \kappa\left(U_{22} U_{31}-U_{21} U_{32}\right) m_{\mu}} \\
h_{32}=\frac{f_{13} h_{12} \kappa m_{e}\left(U_{12} U_{21}-U_{11} U_{22}\right)+m_{2} U_{22}\left(-U_{12} U_{31}+U_{11} U_{32}\right)}{f_{13} \kappa\left(U_{22} U_{31}-U_{21} U_{32}\right) m_{\tau}}, \\
h_{21}=-\frac{\left(U_{12} U_{31}-U_{11} U_{32}\right)\left(f_{13} h_{11} \kappa m_{e}-m_{1} U_{31}\right)}{f_{13} \kappa\left(U_{22} U_{31}-U_{21} U_{32}\right) m_{\mu}}, \\
h_{31}=\frac{f_{13} h_{11} \kappa m_{e}\left(U_{12} U_{21}-U_{11} U_{22}\right)+m_{1} U_{21}\left(-U_{12} U_{31}+U_{11} U_{32}\right)}{f_{13} \kappa\left(U_{22} U_{31}-U_{21} U_{32}\right) m_{\tau}} \\
f_{12}=\frac{f_{13}\left(U_{12} U_{21}-U_{11} U_{22}\right)}{U_{12} U_{31}-U_{11} U_{32}} \\
f_{23}=\frac{f_{13}\left(U_{22} U_{31}-U_{21} U_{32}\right)}{U_{12} U_{31}-U_{11} U_{32}}
\end{gathered}
$$

with $m_{1}=\sqrt{\Delta m_{\mathrm{atm}}^{2}}$ and $m_{2}=\sqrt{\Delta m_{\mathrm{sol}}^{2}+\Delta m_{\mathrm{atm}}^{2}}$.

Inserting the best-fit values [39] for the mixing angles and squared mass differences along with $\delta_{C P}=$ $\pi(0)$ for a $\mathrm{NH}(\mathrm{IH})$, the nonfree $f_{\alpha \beta}$ couplings show a mild hierarchy,

$$
\begin{aligned}
& f_{12} \approx 1.81 f_{13}, \quad f_{23} \approx 2.93 f_{13} \text { for } \mathrm{NH}, \\
& f_{12} \approx-0.88 f_{13}, \quad f_{23} \approx-0.20 f_{13} \text { for } \mathrm{IH} .
\end{aligned}
$$

On the other hand, from the expressions for the nonfree $h_{\beta i}$ Yukawa couplings it is highly expected that those associated with the tau $\left(h_{3 i}\right)$ are suppressed in comparison to those associated with the muon $\left(h_{2 i}\right)$, and these in turn are suppressed in comparison to the free $h_{\beta i}$ parameters $\left(h_{12}\right.$ and $h_{13}$ for $\mathrm{NH}$ and $h_{11}$ and $h_{12}$ for $\left.\mathrm{IH}\right)$. Introducing the benchmark point $m_{s 1}=m_{s 2} / 2=300 \mathrm{GeV}, \sin (2 \varphi)=0.5, f_{13}=0.01$, and $h_{12}=h_{13}=0.1$. $\left(h_{11}=h_{12}=0.1\right)$ along with the previous inputs, the nonfree $h_{\beta i}$ couplings become

$$
\begin{aligned}
& h_{22} \approx-0.00027, \quad h_{23} \approx-0.00027, \quad h_{32} \approx 0.00001, \quad h_{33} \approx 0.00001, \\
& f_{12} \approx 0.00738, \quad f_{23} \approx 0.01761 \quad \text { for } \mathrm{NH}, \\
& h_{22} \approx 0.00243, \quad h_{32} \approx 0.00013, \quad h_{21} \approx 0.00238, \quad h_{31} \approx 0.00012, \\
& f_{12} \approx-0.00876, \quad f_{23} \approx-0.00202 \quad \text { for } \mathrm{IH} .
\end{aligned}
$$

These results illustrate the strong hierarchy present in the $h_{\beta i}$ couplings.

Here a comment is in order concerning the leptonic $C P$ phase, $\delta_{C P}$. Since only one $f_{i j}$ Yukawa coupling-say, $f_{13}$ - can be rendered real, it follows from the expression for $f_{12}$ and $f_{23}$ that the $C P$ symmetry is conserved in the neutrino sector only in the scenario when those couplings are real. That is, such a scenario demands that the Dirac phase necessarily takes the values $\delta_{C P}=0, \pi$. This scenario is in agreement to a large extent with the most recent analysis $[39,40]$ which gave a best fit for the complex phase close to $\delta_{C P}=\pi$ for a $\mathrm{NH}$, whereas $\delta_{C P}=$ 0 is marginally allowed within the $3 \sigma$ range for an $\mathrm{IH}$. This in turn makes such a scenario falsifiable at the upcoming neutrino experiments, such as DUNE [7] and HyperKamiokande [8].

\section{B. LFV processes}

The same Yukawa interactions involved in the neutrino mass generation also induce charged lepton-flavor-violating processes, such as $\ell_{i} \rightarrow \ell_{j} \gamma, \ell_{i} \rightarrow 3 \ell_{j}$, and $\mu-e$ conversion in nuclei. In this model, such processes are generated at the one-loop level and are mediated by the charged scalars $s_{k}^{ \pm}$and neutrinos. The expressions for the branching ratios $\mathcal{B}\left(\ell_{i} \rightarrow \ell_{j} \gamma\right)$ are given by 

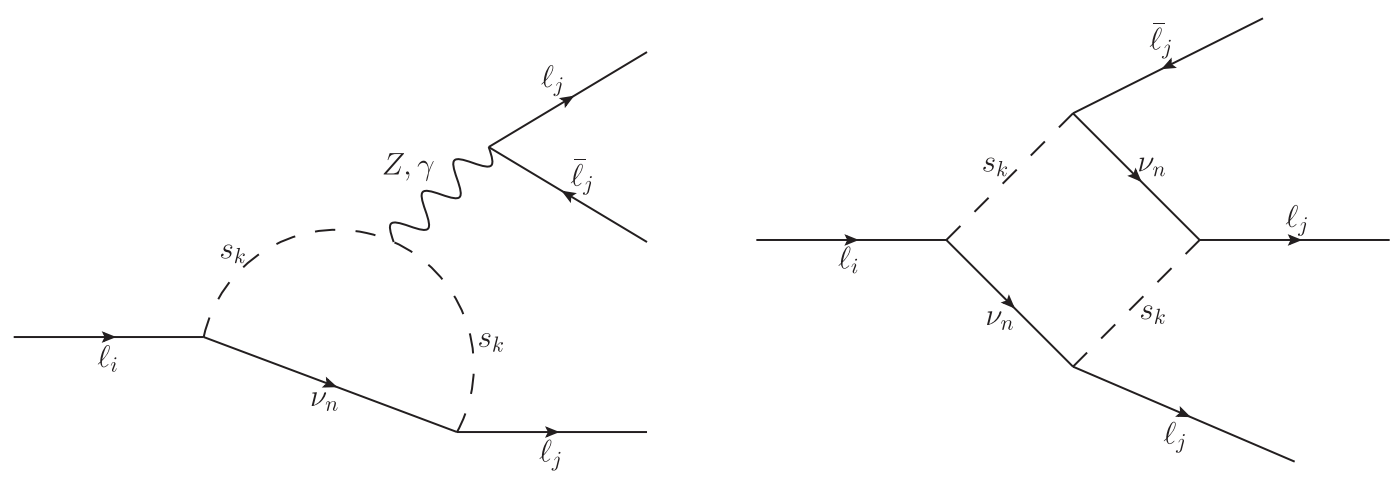

FIG. 2. One-loop Feynman diagrams for the rare decays $\ell_{i} \rightarrow \ell_{j} \bar{\ell}_{j} \ell_{j}$.

$$
\begin{aligned}
& \mathcal{B}(\mu \rightarrow e \gamma)=\frac{\alpha_{e} \operatorname{Br}\left(\mu \rightarrow e \nu_{\mu} \bar{\nu}_{e}\right)}{768 \pi G_{F}^{2}}\left[16 C_{\varphi 12}^{2}\left|f_{13}\right|^{2}\left|f_{23}\right|^{2}+C_{\varphi 21}^{2}\left(\left|h_{12} h_{22}+h_{13} h_{23}\right|^{2}\right)\right], \\
& \mathcal{B}(\tau \rightarrow e \gamma)=\frac{\alpha_{e} \operatorname{Br}\left(\tau \rightarrow e \nu_{\tau} \bar{\nu}_{e}\right)}{768 \pi G_{F}^{2}}\left[16 C_{\varphi 12}^{2}\left|f_{12}\right|^{2}\left|f_{23}\right|^{2}+C_{\varphi 21}^{2}\left(\left|h_{12} h_{32}+h_{13} h_{33}\right|^{2}\right)\right], \\
& \mathcal{B}(\tau \rightarrow \mu \gamma)=\frac{\alpha_{e} \operatorname{Br}\left(\tau \rightarrow \mu \nu_{\tau} \bar{\nu}_{\mu}\right)}{768 \pi G_{F}^{2}}\left[16 C_{\varphi 12}^{2}\left|f_{12}\right|^{2}\left|f_{13}\right|^{2}+C_{\varphi 21}^{2}\left(\left|h_{22} h_{32}+h_{23} h_{33}\right|^{2}\right)\right]
\end{aligned}
$$

for the case of a $\mathrm{NH}$, and

$$
\begin{aligned}
& \mathcal{B}(\mu \rightarrow e \gamma)=\frac{\alpha_{e} \operatorname{Br}\left(\mu \rightarrow e \nu_{\mu} \bar{\nu}_{e}\right)}{768 \pi G_{F}^{2}}\left[16 C_{\varphi 12}^{2}\left|f_{13}\right|^{2}\left|f_{23}\right|^{2}+C_{\varphi 21}^{2}\left(\left|h_{11} h_{21}+h_{12} h_{22}\right|^{2}\right)\right], \\
& \mathcal{B}(\tau \rightarrow e \gamma)=\frac{\alpha_{e} \operatorname{Br}\left(\tau \rightarrow e \nu_{\tau} \bar{\nu}_{e}\right)}{768 \pi G_{F}^{2}}\left[16 C_{\varphi 12}^{2}\left|f_{12}\right|^{2}\left|f_{23}\right|^{2}+C_{\varphi 21}^{2}\left(\left|h_{11} h_{31}+h_{12} h_{32}\right|^{2}\right)\right], \\
& \mathcal{B}(\tau \rightarrow \mu \gamma)=\frac{\alpha_{e} \operatorname{Br}\left(\tau \rightarrow \mu \nu_{\tau} \bar{\nu}_{\mu}\right)}{768 \pi G_{F}^{2}}\left[16 C_{\varphi 12}^{2}\left|f_{12}\right|^{2}\left|f_{13}\right|^{2}+C_{\varphi 21}^{2}\left(\left|h_{21} h_{31}+h_{22} h_{32}\right|^{2}\right)\right]
\end{aligned}
$$

for an IH. Here we have defined $C_{\varphi i j}=\cos \varphi^{2} / m_{s i}^{2}+$ $\sin \varphi^{2} / m_{s j}^{2}$. Notice that, in contrast to neutrino masses, these branching ratios are not suppressed by the scalar mixing angle $\varphi$, as is expected since the rare decays $\ell_{i} \rightarrow$ $\ell_{j} \gamma$ do not depend on the massiveness of neutrinos.

As regards the $\ell_{i} \rightarrow \ell_{j} \bar{\ell}_{j} \ell_{j}$ processes, there exist two main diagrams (see Fig. 2) contributing to the total amplitude: the photon- and $Z$-penguin diagrams (left panel) and the box diagram (right panel). The contribution of the Higgs-penguin diagram is subdominant due to the suppression coming from the Yukawa couplings associated with the first two families of charged leptons (the contribution of the processes involving tau leptons is not negligible, but the corresponding limits are less restrictive), whereas the $Z^{\prime}$-penguin contribution is also subdominant due to the large mass of the $Z^{\prime}$ gauge boson required to surpass the LHC lower bound (see next section). The $\mu-e$ conversion diagrams are obtained when the pair of lepton lines attached to the photon and $Z$ boson in the penguin diagrams are replaced by a pair of light quark lines. ${ }^{6}$ The corresponding amplitudes for the $\ell_{i} \rightarrow \ell_{j} \bar{\ell}_{j} \ell_{j}, \ell_{i} \rightarrow \ell_{j} \bar{\ell}_{k} \ell_{k}$, and $\mu-e$ processes are calculated using the FlavorKit code [41].

Finally, when performing our numerical analysis in Sec. IV we will consider the current experimental bounds and their future expectations shown in Table II.

\section{LHC observables}

LHC searches for additional neutral gauge bosons have delivered stringent constraints on the fraction of the $Z^{\prime}$ mass

${ }^{6}$ Higgs-penguin diagrams are again suppressed, in this case by the Yukawa couplings of light quarks. 
to the gauge coupling, $M_{Z^{\prime}} / g^{\prime}$. The recasting [51] of the latest ATLAS results for the search for dilepton resonances using $139 \mathrm{fb}^{-1}$ [52] for the $U(1)_{B-L}$ model gives $M_{Z^{\prime}} \gtrsim$ $5 \mathrm{TeV}$ for $g^{\prime} \sim 0.1$. Let us recall that the mass of the $Z^{\prime}$ boson is given by $M_{Z^{\prime}}=3 g^{\prime} v_{S}$, which in turn implies $v_{S} \gtrsim 17 \mathrm{TeV}$ for $g^{\prime} \sim 0.1$.

Regarding collider bounds on the charged scalar $s_{1}^{ \pm}$, its main production mechanism at the LHC for small and intermediate Yukawa couplings $\left(f_{\alpha \beta}, h_{\beta i} \lesssim 0.1\right)$ is via Drell-Yan processes. The subsequent decays into a lepton and a neutrino involve precisely these Yukawa interactions. $^{7}$

The expressions for decay width of the processes $\Gamma\left(s_{1} \rightarrow \ell \sum_{i} \nu_{i}\right) \equiv \Gamma\left(s_{1} \rightarrow \ell \nu\right)$ for $\mathrm{NH}$ are given by
TABLE II. Current bounds [42-49] and projected sensitivities [9-11,50] for the charged LFV observables.

\begin{tabular}{lcc}
\hline \hline Observable & Present limit & Future sensitivity \\
\hline $\mathcal{B}(\mu \rightarrow e \gamma)$ & $4.2 \times 10^{-13}$ & $6.3 \times 10^{-14}$ \\
$\mathcal{B}(\tau \rightarrow e \gamma)$ & $3.3 \times 10^{-8}$ & $3 \times 10^{-9}$ \\
$\mathcal{B}(\tau \rightarrow \mu \gamma)$ & $4.4 \times 10^{-8}$ & $3 \times 10^{-9}$ \\
$\mathcal{B}(\mu \rightarrow e e e)$ & $1.0 \times 10^{-12}$ & $10^{-16}$ \\
$\mathcal{B}(\tau \rightarrow e e e)$ & $2.7 \times 10^{-8}$ & $3 \times 10^{-9}$ \\
$\mathcal{B}(\tau \rightarrow \mu \mu \mu)$ & $2.1 \times 10^{-8}$ & $10^{-9}$ \\
$\mathcal{B}(\tau \rightarrow e \mu \mu)$ & $2.7 \times 10^{-8}$ & $\ldots$ \\
$\mathcal{B}(\tau \rightarrow \mu e e)$ & $1.8 \times 10^{-8}$ & $\ldots$ \\
$\mathrm{R}_{\mu \mathrm{e}}(\mathrm{Ti})$ & $4.3 \times 10^{-12}$ & $10^{-18}$ \\
$\mathrm{R}_{\mu \mathrm{e}}(\mathrm{Au})$ & $7.3 \times 10^{-13}$ & $\ldots$ \\
\hline \hline
\end{tabular}

$$
\begin{aligned}
& \Gamma\left(s_{1} \rightarrow e \nu\right)=\frac{m_{s 1}}{16 \pi}\left(4 \cos \varphi^{2}\left(\left|f_{12}\right|^{2}+\left|f_{13}\right|^{2}\right)+\sin \varphi^{2}\left(\left|h_{12}\right|^{2}+\left|h_{13}\right|^{2}\right)\right), \\
& \Gamma\left(s_{1} \rightarrow \mu \nu\right)=\frac{m_{s 1}}{16 \pi}\left(4 \cos \varphi^{2}\left(\left|f_{12}\right|^{2}+\left|f_{23}\right|^{2}\right)+\sin \varphi^{2}\left(\left|h_{22}\right|^{2}+\left|h_{23}\right|^{2}\right)\right), \\
& \Gamma\left(s_{1} \rightarrow \tau \nu\right)=\frac{m_{s 1}}{16 \pi}\left(4 \cos \varphi^{2}\left(\left|f_{13}\right|^{2}+\left|f_{23}\right|^{2}\right)+\sin \varphi^{2}\left(\left|h_{32}\right|^{2}+\left|h_{33}\right|^{2}\right)\right),
\end{aligned}
$$

whereas for IH they read

$$
\begin{aligned}
& \Gamma\left(s_{1} \rightarrow e \nu\right)=\frac{m_{s 1}}{16 \pi}\left(4 \cos \varphi^{2}\left(\left|f_{12}\right|^{2}+\left|f_{13}\right|^{2}\right)+\sin \varphi^{2}\left(\left|h_{11}\right|^{2}+\left|h_{12}\right|^{2}\right)\right), \\
& \Gamma\left(s_{1} \rightarrow \mu \nu\right)=\frac{m_{s 1}}{16 \pi}\left(4 \cos \varphi^{2}\left(\left|f_{12}\right|^{2}+\left|f_{23}\right|^{2}\right)+\sin \varphi^{2}\left(\left|h_{21}\right|^{2}+\left|h_{22}\right|^{2}\right)\right), \\
& \Gamma\left(s_{1} \rightarrow \tau \nu\right)=\frac{m_{s 1}}{16 \pi}\left(4 \cos \varphi^{2}\left(\left|f_{13}\right|^{2}+\left|f_{23}\right|^{2}\right)+\sin \varphi^{2}\left(\left|h_{31}\right|^{2}+\left|h_{32}\right|^{2}\right)\right) .
\end{aligned}
$$

These direct leptonic decays lead to the collider signature of dileptons plus missing transverse momentum since

$$
p p \rightarrow \gamma^{*} / Z^{*} \rightarrow s_{1}^{+} s_{1}^{-} \rightarrow \ell_{i}^{+} \ell_{j}^{-} \nu \nu .
$$

Such a signature is analogous to that of electroweak production of sleptons in the context of simplified supersymmetric scenarios [53,54]. Assuming a 100\% branching ratio into $\ell=e, \mu$ and an integrated luminosity of $139 \mathrm{fb}^{-1}$, ATLAS has set upper limits on the slepton pair production cross sections in such a way that right-handed slepton masses are excluded up to $420 \mathrm{GeV}$ for vanishing

\footnotetext{
${ }^{7}$ Notice that the decays involving gauge bosons are not present.
}

neutralino masses [53]. A recast of the excluded cross section for slepton pair production was done in Ref. [55], which allows to exclude $S U(2)_{L}$-singlet charged scalars decaying into a lepton plus a neutrino with masses up to $200 \mathrm{GeV}$ (or even more in some cases). Moreover, the analysis in Ref. [56] ${ }^{8}$ showed that singly charged scalars with masses below $500 \mathrm{GeV}$ decaying mostly into electrons and muons may be excluded at the high-luminosity phase of the LHC.

On the other hand, single production of charged scalars can also take place (for large Yukawa couplings) through the radiation from a lepton external leg in $s$-channel diagrams featuring a gauge boson $(\gamma, Z$, or $W)$. The collider signatures for these topologies are one, two, or three leptons plus missing transverse momentum. Concerning

\footnotetext{
${ }^{8}$ See Refs. $[57,58]$ for related works.
} 
the final state with three leptons ( $e$ or $\mu$ ), a recasting [59] of the ATLAS trilepton searches [60] excluded charged scalars not decaying into taus with masses $\sim 190 \mathrm{GeV}$ and Yukawa couplings of $\mathcal{O}(1)$.

\section{GENERAL NEUTRINO INTERACTIONS IN THE ZEE DIRAC MODEL}

The $h_{\beta i}$ and $f_{\alpha \beta}$ interactions also induce new effective four-fermion interactions between neutrinos and charged leptons with all of the Lorentz-invariant structures: scalar $(S)$, pseudoscalar $(P)$, vector $(V)$, axial-vector $(A)$, and tensor $(T){ }^{9}$ These new interactions can modify the SM prospect for the neutrino-electron elastic scattering, and therefore be constrained $[32,33,61]$ by using the solar neutrino experiment results, such as those from Borexino [62], TEXONO [63], and CHARM-II [64].

The most general effective Lagrangian that describes the GNI can be cast as ${ }^{10}$

$\mathcal{L}=\frac{G_{F}}{\sqrt{2}} \sum_{a=S, P, V, A, T}\left(\bar{\nu}_{\alpha} \Gamma^{a} \nu_{\beta}\right)\left[\bar{\ell} \Gamma^{a}\left(\epsilon_{\alpha \beta}^{a}+\tilde{\epsilon}_{\alpha \beta}^{a} i^{a} \gamma^{5}\right) \ell\right]$,

where $\quad\left\{\Gamma^{S}, \Gamma^{P}, \Gamma^{V}, \Gamma^{A}, \Gamma^{T}\right\} \equiv\left\{I, i \gamma^{5}, \gamma^{\mu}, \gamma^{\mu} \gamma^{5}, \sigma^{\mu \nu}\right\}$, $\left\{i^{S}, i^{P}, i^{V}, i^{A}, i^{T}\right\} \equiv\{i, i, 1,1, i\}$, and $G_{F}$ is the Fermi constant. The $3 \times 3$ matrices $\epsilon$ and $\tilde{\epsilon}$-which are assumed to be real-parametrize the departures of the SM result.

On the other hand, the effective four-fermion Lagrangian for GNI contributions in the present model is given by

$$
\begin{aligned}
\mathcal{L}_{\text {eff }}= & -2 f_{\alpha \rho} f_{\beta \sigma}^{*}\left[\left(\overline{\ell_{\beta}} \gamma^{\mu} P_{L} \ell_{\alpha}\right)\left(\overline{\nu_{\sigma}} \gamma_{\mu} P_{L} \nu_{\rho}\right)\right] C_{\phi 12}-\frac{1}{2} h_{\alpha \rho} h_{\beta \sigma}^{*}\left[\left(\overline{\ell_{\beta}} \gamma^{\mu} P_{R} \ell_{\alpha}\right)\left(\overline{\nu_{\sigma}} \gamma_{\mu} P_{R} \nu_{\rho}\right)\right] C_{\phi 21} \\
& +f_{\alpha \rho} h_{\beta \sigma}^{*}\left[\left(\overline{\ell_{\beta}} P_{L} \ell_{\alpha}\right)\left(\overline{\nu_{\sigma}} P_{L} \nu_{\rho}\right)-\frac{1}{4}\left(\overline{\ell_{\beta}} \sigma^{\mu \nu} P_{L} \ell_{\alpha}\right)\left(\overline{\nu_{\sigma}} \sigma_{\mu \nu} P_{L} \nu_{\rho}\right)\right] D_{\phi 12} \\
& +f_{\beta \sigma}^{*} h_{\alpha \rho}\left[\left(\overline{\ell_{\beta}} P_{R} \ell_{\alpha}\right)\left(\overline{\nu_{\sigma}} P_{R} \nu_{\rho}\right)-\frac{1}{4}\left(\overline{\ell_{\beta}} \sigma^{\mu \nu} P_{R} \ell_{\alpha}\right)\left(\overline{\nu_{\sigma}} \sigma_{\mu \nu} P_{R} \nu_{\rho}\right)\right] D_{\phi 12},
\end{aligned}
$$

where $D_{\varphi i j}=\cos \varphi \sin \varphi\left(1 / m_{s j}^{2}-1 / m_{s i}^{2}\right)$. It follows that the factors in the second and third lines in the previous expression become suppressed for a small scalar mixing angle, while the two factors in the first line are suppressed for small Yukawa couplings, respectively.

For electron neutrinos $\sigma=\rho=e$ the corresponding GNI read

$$
\begin{gathered}
\epsilon_{e e}^{V}=\tilde{\epsilon}_{e e}^{V}=\epsilon_{e e}^{A}=\tilde{\epsilon}_{e e}^{A}=-\frac{\sqrt{2}}{8 G_{F}}\left|h_{11}\right|^{2} C_{\varphi 21}, \\
\epsilon_{e e}^{S}=\tilde{\epsilon}_{e e}^{S}=\epsilon_{e e}^{P}=\tilde{\epsilon}_{e e}^{P}=\epsilon_{e e}^{T}=\tilde{\epsilon}_{e e}^{T}=0 .
\end{gathered}
$$

Notice that all GNI associated with electron neutrinos for the $\mathrm{NH}$ are zero since $h_{i 1}=0$. For muon neutrinos $\sigma=$ $\rho=\mu$ it follows that

$$
\begin{aligned}
& \epsilon_{\mu \mu}^{V}=\epsilon_{\mu \mu}^{A}=-\frac{\sqrt{2}}{8 G_{F}}\left(\left|h_{12}\right|^{2} C_{\phi 21}+4\left|f_{12}\right|^{2} C_{\varphi 12}\right), \\
& \tilde{\epsilon}_{\mu \mu}^{V}=\tilde{\epsilon}_{\mu \mu}^{A}=-\frac{\sqrt{2}}{8 G_{F}}\left(\left|h_{12}\right|^{2} C_{\varphi 21}-4\left|f_{12}\right|^{2} C_{\varphi 12}\right),
\end{aligned}
$$

\footnotetext{
${ }^{9}$ There are also effective four-fermion interactions mediated by the $Z^{\prime}$ boson; however, they turn out to be heavily suppressed by $M_{Z^{\prime}}$.

${ }^{10} \mathrm{We}$ closely follow the notation introduced in Ref. [32].
}

$$
\begin{gathered}
\epsilon_{\mu \mu}^{S}=-\epsilon_{\mu \mu}^{P}=-4 \epsilon_{\mu \mu}^{T}=\frac{\sqrt{2} D_{\varphi 12}}{4 G_{F}}\left(f_{12} h_{12}^{*}+f_{12}^{*} h_{12}\right), \\
\tilde{\epsilon}_{\mu \mu}^{S}=-\tilde{\epsilon}_{\mu \mu}^{P}=-4 \tilde{\epsilon}_{\mu \mu}^{T}=\frac{i \sqrt{2} D_{\phi 12}}{4 G_{F}}\left(f_{12} h_{12}^{*}-f_{12}^{*} h_{12}\right) .
\end{gathered}
$$

For the case of tau neutrinos $\sigma=\rho=\tau$ the GNI are

$$
\epsilon_{\tau \tau}^{V}=\epsilon_{\tau \tau}^{A}=-\frac{\sqrt{2}}{8 G_{F}}\left(\left|h_{13}\right|^{2} C_{\varphi 21}+4\left|f_{13}\right|^{2} C_{\varphi 12}\right),
$$

TABLE III. Current bounds and projected sensitivities for GNI [32].

\begin{tabular}{lcc}
\hline \hline GNI & Current bound & Projected sensitivity \\
\hline$\epsilon_{e e}^{V}$ & {$[-0.12,0.08]$} & {$[-0.016,0.016]$} \\
$\epsilon_{e e}^{A}$ & {$[-0.13,0.07]$} & {$[-0.016,0.016]$} \\
$\tilde{\epsilon}_{e e}^{V}$ & {$[-0.07,0.13]$} & {$[-0.016,0.016]$} \\
$\tilde{\epsilon}_{e e}^{A}$ & {$[-0.08,0.13]$} & {$[-0.016,0.016]$} \\
$\epsilon_{\mu \mu}^{V} / \epsilon_{\tau \tau}^{V}$ & {$[-0.22,0.08]$} & {$[-0.1,0.1]$} \\
$\epsilon_{\mu \mu}^{A} / \epsilon_{\tau \tau}^{A}$ & {$[-0.08,0.08]$} & {$[-0.03,0.03]$} \\
$\tilde{\epsilon}_{\mu \mu}^{V} / \tilde{\epsilon}_{\tau \tau}^{V}$ & {$[-0.09,0.08]$} & {$[-0.04,0.04]$} \\
$\tilde{\epsilon}_{\mu \mu}^{A} / \tilde{\epsilon}_{\tau \tau}^{A}$ & {$[-0.90,0.22]$} & {$[-0.1,0.1]$} \\
$\epsilon_{\mu \mu}^{S} / \epsilon_{\tau \tau}^{S}$ & {$[-0.83,0.83]$} & {$[-0.5,0.5]$} \\
$\epsilon_{\mu \mu}^{P} / \epsilon_{\tau \tau}^{P}$ & {$[-0.83,0.83]$} & {$[-1.22,1.22]$} \\
$\epsilon_{\mu \mu}^{T} / \epsilon_{\tau \tau}^{T}$ & {$[-0.15,0.15]$} & {$[-0.1,0.1]$} \\
\hline \hline
\end{tabular}




$$
\begin{array}{r}
\tilde{\epsilon}_{\tau \tau}^{V}=\tilde{\epsilon}_{\tau \tau}^{A}=-\frac{\sqrt{2}}{8 G_{F}}\left(\left|h_{13}\right|^{2} C_{\varphi 21}-4\left|f_{13}\right|^{2} C_{\varphi 12}\right), \\
\epsilon_{\tau \tau}^{S}=-\epsilon_{\tau \tau}^{P}=-4 \epsilon_{\tau \tau}^{T}=\frac{\sqrt{2} D_{\varphi 12}}{4 G_{F}}\left(f_{13} h_{13}^{*}+f_{13}^{*} h_{13}\right), \\
\tilde{\epsilon}_{\tau \tau}^{S}=-\tilde{\epsilon}_{\tau \tau}^{P}=-4 \tilde{\epsilon}_{\tau \tau}^{T}=\frac{i \sqrt{2} D_{\phi 12}}{4 G_{F}}\left(f_{13} h_{13}^{*}-f_{13}^{*} h_{13}\right) .
\end{array}
$$

Because $h_{i 3}=0$ in an inverted neutrino mass ordering, these GNI reduce to

$$
\begin{gathered}
\epsilon_{\tau \tau}^{V}=\epsilon_{\tau \tau}^{A}=-\tilde{\epsilon}_{\tau \tau}^{V}=-\tilde{\epsilon}_{\tau \tau}^{A}=-\frac{\sqrt{2}}{2 G_{F}}\left|f_{13}\right|^{2} C_{\varphi 12}, \\
\epsilon_{\tau \tau}^{S}=\epsilon_{\tau \tau}^{P}=\epsilon_{\tau \tau}^{T}=\tilde{\epsilon}_{\tau \tau}^{S}=\tilde{\epsilon}_{\tau \tau}^{P}=\tilde{\epsilon}_{\tau \tau}^{T}=0 .
\end{gathered}
$$

By using the Borexino results, the authors of Ref. [32] derived $90 \%$ C.L. constraints on general neutrino interactions for all neutrino flavors. The most constraining
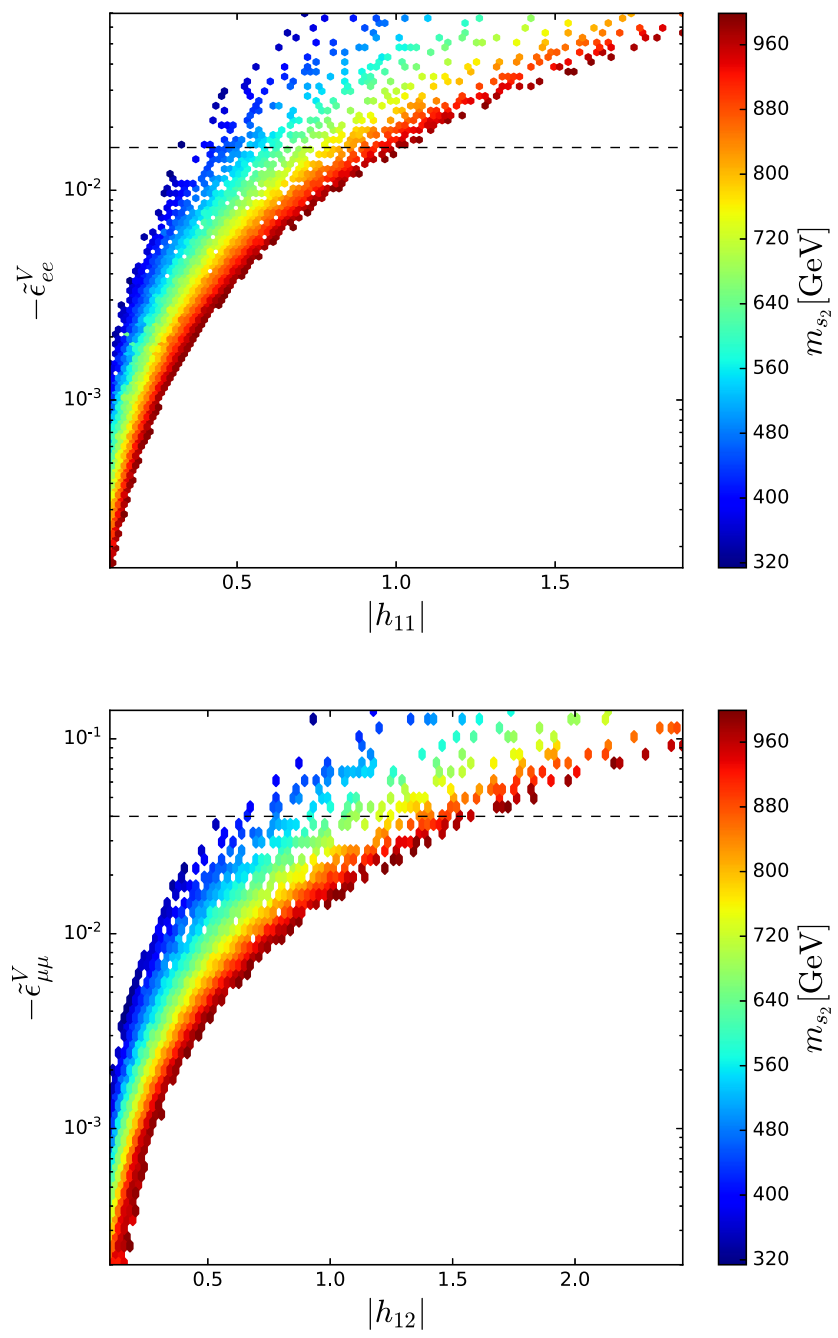

bounds that apply to the current model are displayed in Table III.

\section{RESULTS AND DISCUSSION}

We have performed a random scan for each neutrino mass ordering, varying just a subset of the free parameters of the model with the aim of making the analysis simpler. The set of free parameters of the model relevant for our analysis has been varied as

$$
\begin{aligned}
10^{-5} & \leq\left|f_{13}\right| \leq 3, \\
0.1 & \leq\left|h_{12}, h_{13}\left(h_{11}, h_{12}\right)\right| \leq 3, \\
10^{-6} & \leq \varphi \leq \pi / 2, \\
80 \mathrm{GeV} & \leq m_{s 1} \leq 500 \mathrm{GeV}, \quad m_{s_{2}}=\left[m_{s_{1}}, 1000 \mathrm{GeV}\right] .
\end{aligned}
$$

The magnitude of the nonfree Yukawa couplings are restricted to be within the range $\left[10^{-5}, 3\right]$, and the other
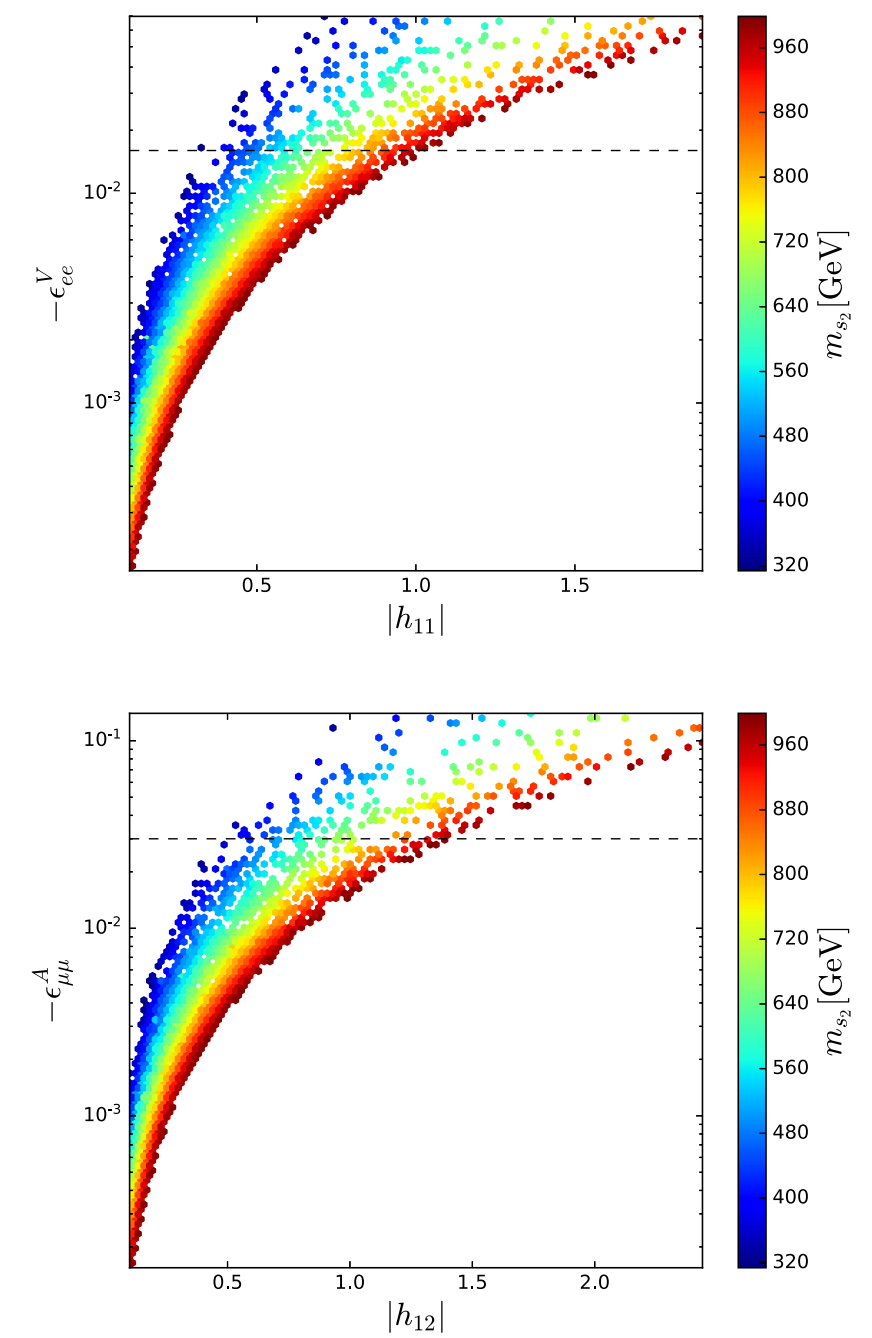

FIG. 3. General neutrino interactions for the IH case. 

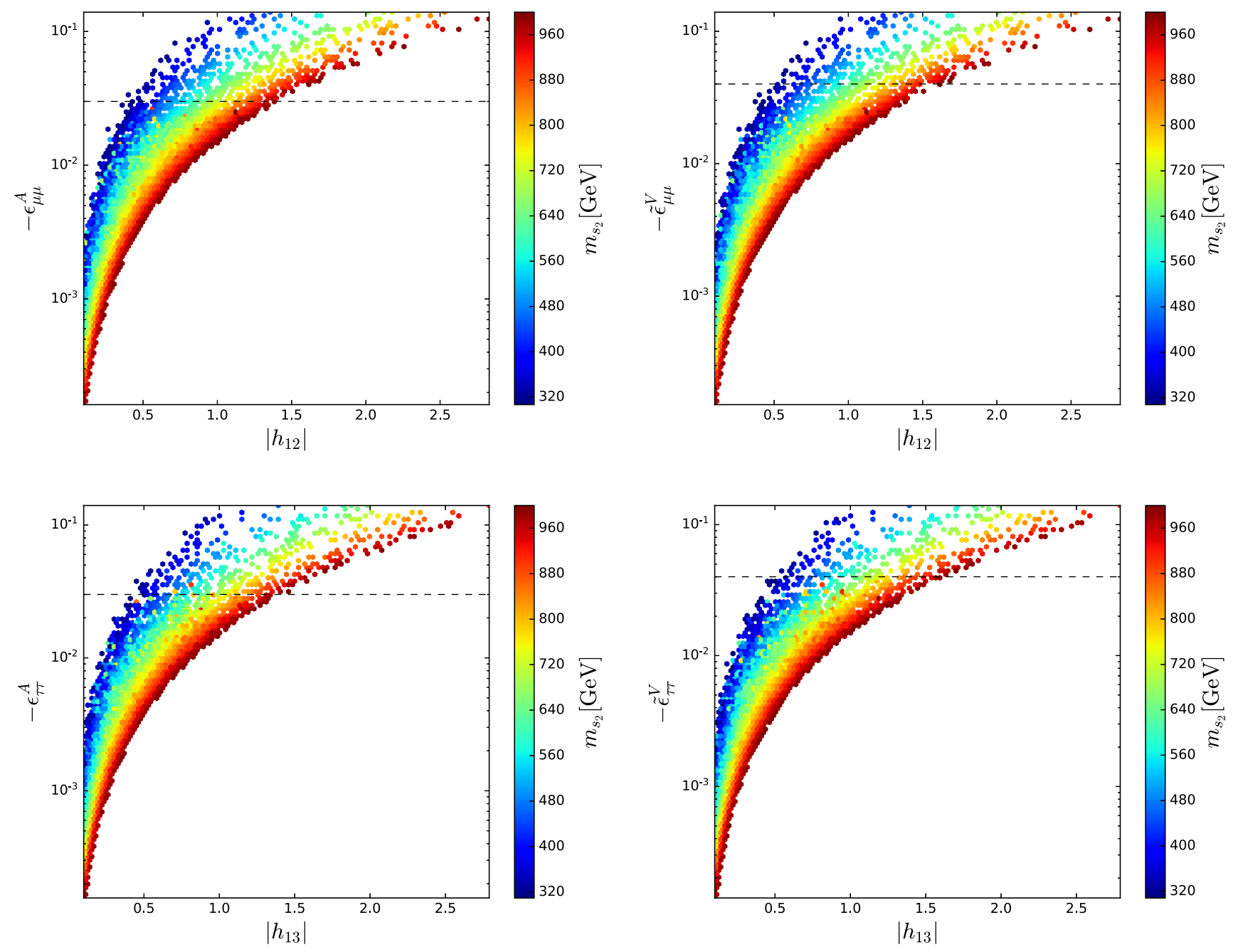

FIG. 4. General neutrino interactions for the NH case.

parameters of the scalar potential were fixed as $\lambda_{i}=10^{-4}$, $i=1, \ldots, 8$. The parameters of the new gauge boson are set to $M_{Z^{\prime}}=6 \mathrm{TeV}$ and $g^{\prime}=0.5$ in such a way that $v_{S}=4 \mathrm{TeV} .{ }^{11}$ In our numerical analysis, all of the viable benchmark points satisfy the current neutrino oscillation data within the $3 \sigma$ level [40], both for inverted and normal hierarchies, with $\delta_{C P}=\pi(0)$ for a NH (IH). Likewise, they satisfy the LFV upper bounds reported in Table II and the GNI constraints in Table III. Finally, we imposed the collider bounds on the charged scalar coming from searches for final states with an oppositely charged lepton pair $\left(e^{-} e^{+}\right.$ and $\mu^{-} \mu^{+}$) and missing transverse energy [55]. In order to obtain the particle spectrum and low-energy observables, we have used SPheno [65,66] and the FlavorKit [41] of SARAH $[67,68]$.

In Fig. 3 (Fig. 4) we display the results for the general neutrino interactions assuming an inverted (normal)

\footnotetext{
${ }^{11}$ With these values it follows that $\mu_{3}<200 \mathrm{GeV}$, in order to obtain charged scalars below the $\mathrm{TeV}$ mass scale.
}

neutrino mass hierarchy, and the future sensitivity (dashed line) that may be reached in future solar neutrino experiments [32], with the color bar denoting the mass of the heaviest charged scalar. Only the results for the parameters $\tilde{\epsilon}_{e e}^{V}, \tilde{\epsilon}_{\mu \mu}^{V}, \epsilon_{e e}^{V}$, and $\epsilon_{\mu \mu}^{A}\left(\tilde{\epsilon}_{\mu \mu}^{V}, \tilde{\epsilon}_{\tau \tau}^{V}, \epsilon_{\mu \mu}^{A}\right.$, and $\left.\epsilon_{\tau \tau}^{A}\right)$ are displayed since they are the ones with the most appreciable restrictions on future experiments. As is expected, the heavier the $s_{2}^{ \pm}$, the lower the intensity of the general neutrino interactions.

The correlation between the most constrained LFV processes, namely, $\mathcal{B}(\mu \rightarrow e \gamma), \mathcal{B}(\mu \rightarrow e \gamma)$, and $\mu-e$ conversion, is shown in the top panels of Fig. 5. The corresponding prospects for the future sensitivities (dashed and dotted lines) show that a large portion of the parameter space will be explored. On the other hand, the current limits impose mild restrictions on the Yukawa couplings; in particular, $\mathcal{B}(\mu \rightarrow e \gamma)$ establishes that $f_{13}$ is restricted to values less than 0.04 . Furthermore, future searches for these processes restrict the value of $f_{13}$ to around $10^{-3}$. Finally, Fig. 6 shows the results of the search for charged scalars at 

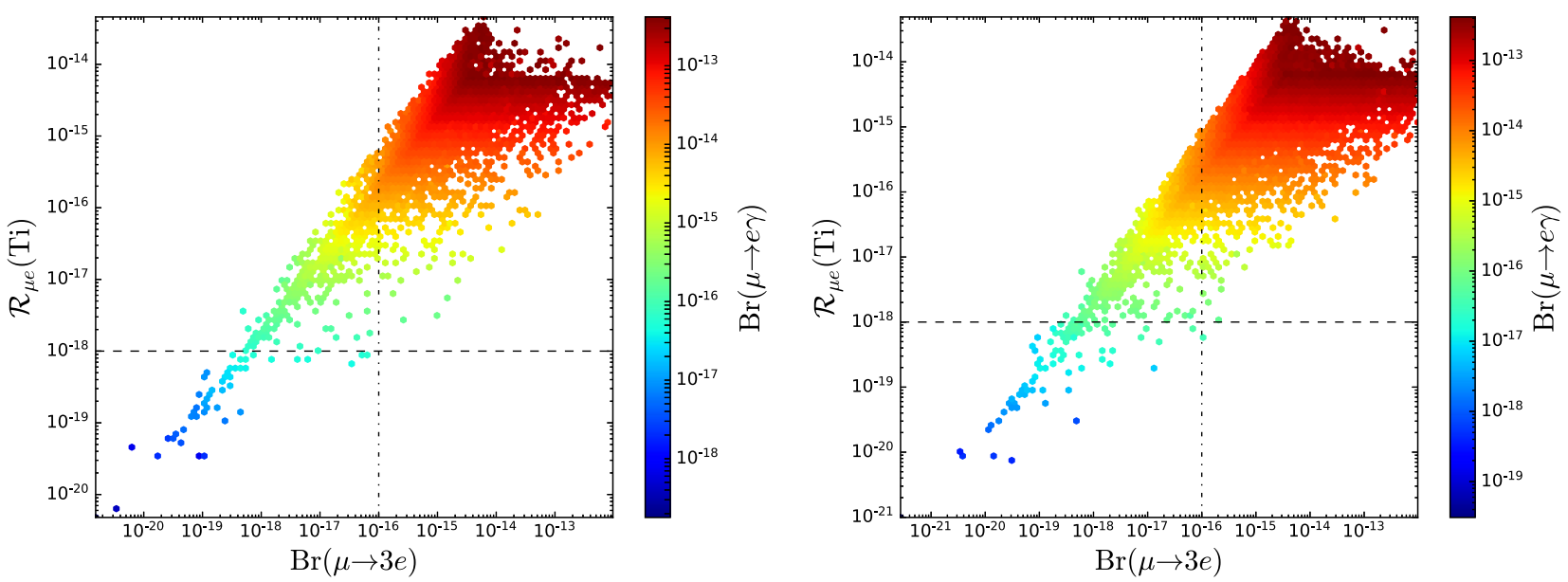

FIG. 5. LFV processes for IH (left) and NH (right). The dotted and dashed lines represent the sensitivity limit expected for the future searches for $\mathcal{R}_{\mu e}(\mathrm{Ti})$ and $\mathcal{B}(\mu \rightarrow 3 e)$, respectively.
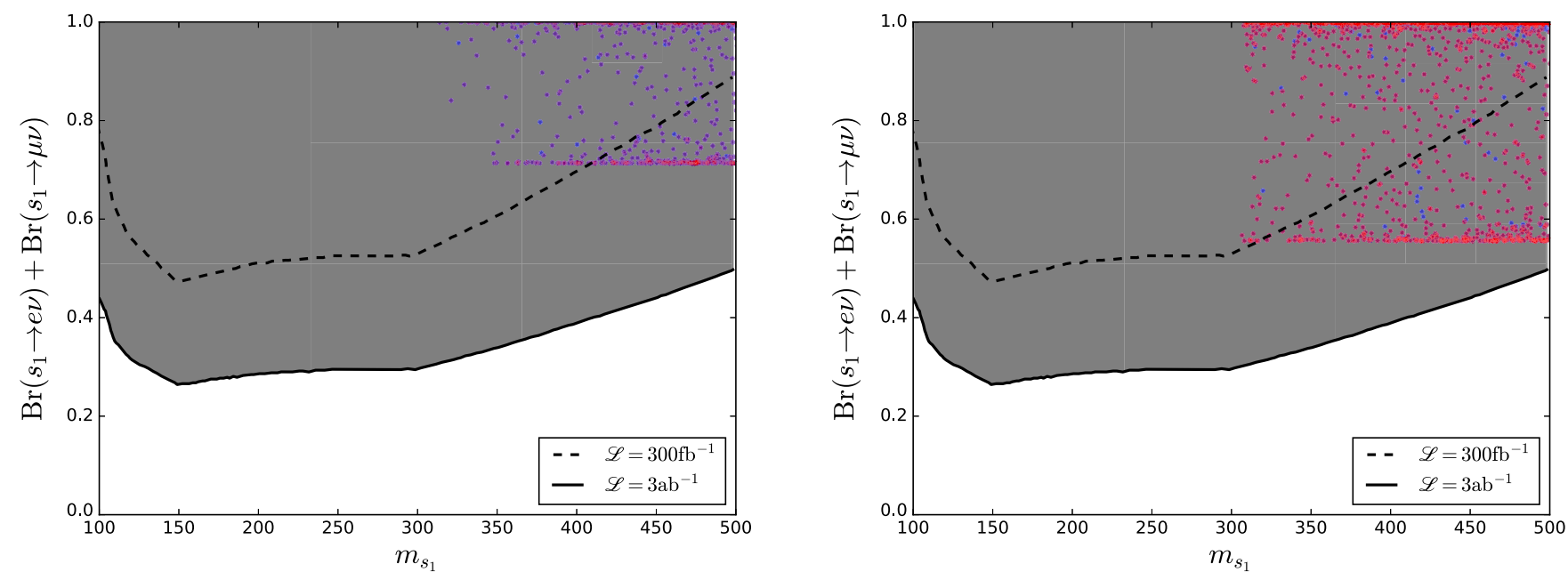

FIG. 6. Projected exclusion reach in the decay branching ratio of $s_{1}^{ \pm}$into electrons and muons with luminosities of $300 \mathrm{fb}^{-1}$ (dashed line) and $3 \mathrm{ab}^{-1}$ (solid line) for IH (left) and NH (right). The red dots are within the future sensitivity of the LFV experiments (see Fig. 5).

the LHC. The current limits impose that the mass of the lightest scalar must be greater than $\sim 260$ and $\sim 300 \mathrm{GeV}$ for a normal and inverted hierarchy, respectively [55]. The dashed line shows the future exclusion limits for an integrated luminosity of $300 \mathrm{fb}^{-1}$ and the solid line for $3 \mathrm{ab}^{-1}$ [56]. Notice that in principle it is possible to explore the whole parameter space in the high-luminosity phase of the LHC.

Since the relevant GNI parameters and the decay rates for $s_{1} \rightarrow \ell \nu$ depend only on the magnitude of the Yukawa couplings, it is understandable to expect that the above results hold in the scenario where the Yukawa couplings are complex. In order to check this, we have performed an additional scan for the scenario where the Dirac $C P$ phase varies within the $3 \sigma$ level [40]. The results from this scan concerning the magnitude of the GNI interactions and the reach of LHC searches do not differ from those obtained in the scenario with $C P$ conservation. Moreover, the LFV observables continue to exhibit a strong correlation as the one displayed in Fig. 5.

\section{SUMMARY}

The simplest model leading to Dirac neutrino masses at the one-loop level was obtained by adding a pair of charged scalars to the SM along with three right-handed neutrinos. In this work, we have assumed that the Diracness of the neutrinos is protected by only one extra $U(1)_{B-L}$ gauge symmetry, with the anomalies canceled by the SM leptons and the three right-handed neutrinos. We studied the interesting phenomenological features of the model, such as the neutrino mass generation, charged LFV processes, and signatures at the LHC associated with the charged scalars and the new $B-L$ gauge boson. Furthermore, we reported the expressions for the general neutrino-electron 
interactions and identified the regions of parameter space that may be explored in future solar neutrino experiments. Remarkably, future searches for charged scalars at the LHC will probe the entire parameter space considered in this analysis.

\section{ACKNOWLEDGMENTS}

The work of D. R. and O.Z. is partially supported by Sostenibilidad-UdeA and UdeA/CODI Grants No. 201716286 and No. 2020-33177.
[1] Y. Fukuda et al. (Super-Kamiokande Collaboration), Evidence for Oscillation of Atmospheric Neutrinos, Phys. Rev. Lett. 81, 1562 (1998).

[2] Q. R. Ahmad et al. (SNO Collaboration), Direct Evidence for Neutrino Flavor Transformation from Neutral Current Interactions in the Sudbury Neutrino Observatory, Phys. Rev. Lett. 89, 011301 (2002).

[3] M. J. Dolinski, A. W. P. Poon, and W. Rodejohann, Neutrinoless double-beta decay: Status and prospects, Annu. Rev. Nucl. Part. Sci. 69, 219 (2019).

[4] M. Lindner, M. Platscher, and F. S. Queiroz, A call for new physics: The muon anomalous magnetic moment and lepton flavor violation, Phys. Rep. 731, 1 (2018).

[5] L. Calibbi and G. Signorelli, Charged lepton flavour violation: An experimental and theoretical introduction, Riv. Nuovo Cimento 41, 1 (2018).

[6] Z. Djurcic et al. (JUNO Collaboration), JUNO conceptual design report, arXiv:1508.07166.

[7] R. Acciarri et al. (DUNE Collaboration), Long-baseline neutrino facility (LBNF) and deep underground neutrino experiment (DUNE): Conceptual design report, volume 2: The physics program for DUNE at LBNF, arXiv:1512 .06148 .

[8] K. Abe et al. (Hyper-Kamiokande Collaboration), HyperKamiokande design report, arXiv:1805.04163.

[9] A. M. Baldini et al., MEG upgrade proposal, arXiv:1301 .7225 .

[10] T. Aushev et al., Physics at super B factory, arXiv:1002 .5012 .

[11] R. J. Abrams et al. (Mu2e Collaboration), Mu2e conceptual design report, arXiv:1211.7019.

[12] A. Das and N. Okada, Bounds on heavy Majorana neutrinos in type-I seesaw and implications for collider searches, Phys. Lett. B 774, 32 (2017).

[13] A. Das, P. S. B. Dev, and N. Okada, Long-lived TeV-scale right-handed neutrino production at the LHC in gauged $U(1)_{X}$ model, Phys. Lett. B 799, 135052 (2019).

[14] A. Das and N. Okada, Inverse seesaw neutrino signatures at the LHC and ILC, Phys. Rev. D 88, 113001 (2013).

[15] P. Minkowski, $\mu \rightarrow e \gamma$ at a rate of one out of $10^{9}$ muon decays? Phys. Lett. 67B, 421 (1977).

[16] T. Yanagida, Horizontal gauge symmetry and masses of neutrinos, Conf. Proc. C7902131, 95 (1979).

[17] M. Gell-Mann, P. Ramond, and R. Slansky, Complex spinors and unified theories, Conf. Proc. C790927, 315 (1979).

[18] R. N. Mohapatra and G. Senjanovic, Neutrino Mass and Spontaneous Parity Nonconservation, Phys. Rev. Lett. 44, 912 (1980).
[19] A. Davidson, $B-L$ as the fourth color within an $\mathrm{SU}(2)_{L} \times$ $\mathrm{U}(1)_{R} \times \mathrm{U}(1)$ model, Phys. Rev. D 20, 776 (1979).

[20] J. Calle, D. Restrepo, C. E. Yaguna, and Ó. Zapata, Minimal radiative Dirac neutrino mass models, Phys. Rev. D 99, 075008 (2019).

[21] C. Bonilla, S. Centelles-Chuliá, R. Cepedello, E. Peinado, and R. Srivastava, Dark matter stability and Dirac neutrinos using only Standard Model symmetries, Phys. Rev. D 101, 033011 (2020).

[22] S. Saad, Simplest radiative Dirac neutrino mass models, Nucl. Phys. B943, 114636 (2019).

[23] Y. Cai, J. Herrero-García, M. A. Schmidt, A. Vicente, and R. R. Volkas, From the trees to the forest: A review of radiative neutrino mass models, Front. Phys. 5, 63 (2017).

[24] S. Nasri and S. Moussa, Model for small neutrino masses at the TeV scale, Mod. Phys. Lett. A 17, 771 (2002).

[25] S. Kanemura, T. Nabeshima, and H. Sugiyama, Neutrino masses from loop-induced Dirac Yukawa couplings, Phys. Lett. B 703, 66 (2011).

[26] S. Jana, P. K. Vishnu, and S. Saad, Minimal dirac neutrino mass models from $U(1)_{\mathrm{R}}$ gauge symmetry and leftright asymmetry at colliders, Eur. Phys. J. C 79, 916 (2019).

[27] C.-Y. Yao and G.-J. Ding, Systematic analysis of Dirac neutrino masses at dimension five, Phys. Rev. D 97, 095042 (2018).

[28] S. Jana, P. K. Vishnu, and S. Saad, Minimal realizations of Dirac neutrino mass from generic one-loop and two-loop topologies at $d=5$, J. Cosmol. Astropart. Phys. 04 (2020) 018.

[29] A. Zee, A theory of lepton number violation, neutrino majorana mass, and oscillation, Phys. Lett. B 93, 389 (1980); Erratum, Phys. Lett. B 95, 461 (1980).

[30] S. T. Petcov, Remarks on the Zee model of neutrino mixing ( $\mu \rightarrow e+\gamma, \nu_{H \rightarrow \nu_{L}+\gamma}$, etc.), Phys. Lett. 115B, 401 (1982).

[31] S. Bergmann, Y. Grossman, and E. Nardi, Neutrino propagation in matter with general interactions, Phys. Rev. D 60, 093008 (1999).

[32] A. N. Khan, W. Rodejohann, and X.-J. Xu, Borexino and general neutrino interactions, Phys. Rev. D 101, 055047 (2020).

[33] I. Bischer and W. Rodejohann, General neutrino interactions from an effective field theory perspective, Nucl. Phys. B947, 114746 (2019).

[34] R. Jinno and M. Takimoto, Probing a classically conformal B-L model with gravitational waves, Phys. Rev. D 95, 015020 (2017). 
[35] W. Chao, W.-F. Cui, H.-K. Guo, and J. Shu, Gravitational wave imprint of new symmetry breaking, Chin. Phys. C 44, 123102 (2020).

[36] C. Marzo, L. Marzola, and V. Vaskonen, Phase transition and vacuum stability in the classically conformal B-L model, Eur. Phys. J. C 79, 601 (2019).

[37] J. C. Montero and V. Pleitez, Gauging U(1) symmetries and the number of right-handed neutrinos, Phys. Lett. B 675, 64 (2009).

[38] E. Ma and R. Srivastava, Dirac or inverse seesaw neutrino masses with $B-L$ gauge symmetry and $S_{3}$ flavor symmetry, Phys. Lett. B 741, 217 (2015).

[39] P. F. de Salas, D. V. Forero, S. Gariazzo, P. Martínez-Miravé, O. Mena, C. A. Ternes, M. Tórtola, and J. W. F. Valle, 2020 Global reassessment of the neutrino oscillation picture, arXiv:2006.11237.

[40] I. Esteban, M. C. Gonzalez-Garcia, M. Maltoni, T. Schwetz, and A. Zhou, The fate of hints: Updated global analysis of three-flavor neutrino oscillations, J. High Energy Phys. 09 (2020) 178.

[41] W. Porod, F. Staub, and A. Vicente, A flavor kit for BSM models, Eur. Phys. J. C 74, 2992 (2014).

[42] A. M. Baldini et al. (MEG Collaboration), Search for the lepton flavour violating decay $\mu^{+} \rightarrow \mathrm{e}^{+} \gamma$ with the full dataset of the MEG experiment, Eur. Phys. J. C 76, 434 (2016).

[43] B. Aubert et al. (BABAR Collaboration), Searches for Lepton Flavor Violation in the Decays $\tau^{ \pm} \rightarrow \mathrm{e}^{ \pm} \gamma$ and $\tau^{ \pm} \rightarrow \mu^{ \pm} \gamma$, Phys. Rev. Lett. 104, 021802 (2010).

[44] M. Bona et al. (SuperB Collaboration), SuperB: A highluminosity asymmetric e+ e- super flavor factory. Conceptual design report, arXiv:0709.0451.

[45] Y. Miyazaki et al. (Belle Collaboration), Search for leptonflavor-violating and lepton-number-violating $\tau \rightarrow \ell h h^{\prime}$ decay modes, Phys. Lett. B 719, 346 (2013).

[46] U. Bellgardt et al. (SINDRUM Collaboration), Search for the decay $\mu^{+} \rightarrow e^{+} e^{+} e^{-}$, Nucl. Phys. B299, 1 (1988).

[47] K. Hayasaka et al., Search for lepton flavor violating Tau decays into three leptons with 719 million produced $\tau^{+} \tau^{-}$ pairs, Phys. Lett. B 687, 139 (2010).

[48] C. Dohmen et al. (SINDRUM II Collaboration), Test of lepton flavor conservation in $\mu \rightarrow e$ conversion on titanium, Phys. Lett. B 317, 631 (1993).

[49] W. H. Bertl et al. (SINDRUM II Collaboration), A search for muon to electron conversion in muonic gold, Eur. Phys. J. C 47, 337 (2006).

[50] A. Blondel et al., Research proposal for an experiment to search for the decay $\mu \rightarrow e e e$, arXiv:1301.6113.

[51] C.-W. Chiang, G. Cottin, A. Das, and S. Mandal, Displaced heavy neutrinos from $Z^{\prime}$ decays at the LHC, J. High Energy Phys. 12 (2019) 070.

[52] G. Aad et al. (ATLAS Collaboration), Search for high-mass dilepton resonances using $139 \mathrm{fb}^{-1}$ of $p p$ collision data collected at $\sqrt{s}=13 \mathrm{TeV}$ with the ATLAS detector, Phys. Lett. B 796, 68 (2019).
[53] G. Aad et al. (ATLAS Collaboration), Search for electroweak production of charginos and sleptons decaying into final states with two leptons and missing transverse momentum in $\sqrt{s}=13 \mathrm{TeV} p p$ collisions using the ATLAS detector, Eur. Phys. J. C 80, 123 (2020).

[54] A. M. Sirunyan et al. (CMS Collaboration), Search for supersymmetry in final states with two oppositely charged same-flavor leptons and missing transverse momentum in proton-proton collisions at $\sqrt{s}=13 \mathrm{TeV}$, J. High Energy Phys. 04 (2021) 123.

[55] A. Crivellin, F. Kirk, C. A. Manzari, and L. Panizzi, Searching for lepton flavour (universality) violation and collider signals from a singly-charged scalar singlet, Phys. Rev. D 103, 073002 (2021).

[56] J. Alcaide and N. I. Mileo, LHC sensitivity to singlycharged scalars decaying into electrons and muons, Phys. Rev. D 102, 075030 (2020).

[57] Q.-H. Cao, G. Li, K.-P. Xie, and J. Zhang, Searching for weak singlet charged scalar at the Large Hadron Collider, Phys. Rev. D 97, 115036 (2018).

[58] K. S. Babu, P. S. B. Dev, S. Jana, and A. Thapa, Nonstandard interactions in radiative neutrino mass models, J. High Energy Phys. 03 (2020) 006.

[59] J. Alcaide, M. Chala, and A. Santamaria, LHC signals of radiatively-induced neutrino masses and implications for the Zee-Babu model, Phys. Lett. B 779, 107 (2018).

[60] G. Aad et al. (ATLAS Collaboration), Search for new phenomena in events with three or more charged leptons in $p p$ collisions at $\sqrt{s}=8 \mathrm{TeV}$ with the ATLAS detector, J. High Energy Phys. 08 (2015) 138.

[61] W. Rodejohann, X.-J. Xu, and C. E. Yaguna, Distinguishing between Dirac and Majorana neutrinos in the presence of general interactions, J. High Energy Phys. 05 (2017) 024.

[62] G. Bellini et al., Precision Measurement of the 7Be Solar Neutrino Interaction Rate in Borexino, Phys. Rev. Lett. 107, 141302 (2011).

[63] M. Deniz et al. (TEXONO Collaboration), Measurement of $\mathrm{Nu}(\mathrm{e})$-bar -electron scattering cross-section with a CsI(Tl) scintillating crystal array at the Kuo-Sheng nuclear power reactor, Phys. Rev. D 81, 072001 (2010).

[64] P. Vilain et al. (CHARM-II Collaboration), Precision measurement of electroweak parameters from the scattering of muon-neutrinos on electrons, Phys. Lett. B 335, 246 (1994).

[65] W. Porod, SPheno, a program for calculating supersymmetric spectra, SUSY particle decays and SUSY particle production at e+ e- colliders, Comput. Phys. Commun. 153, 275 (2003).

[66] W. Porod and F. Staub, SPheno 3.1: Extensions including flavour, CP-phases and models beyond the MSSM, Comput. Phys. Commun. 183, 2458 (2012).

[67] F. Staub, SARAH 4: A tool for (not only SUSY) model builders, Comput. Phys. Commun. 185, 1773 (2014).

[68] F. Staub, Exploring new models in all detail with SARAH, Adv. High Energy Phys. 2015, 840780 (2015). 